\title{
Herramientas a considerar para mantener o aumentar la productividad de la fuerza de ventas
}

\section{Tools to consider to maintain or increase the productivity of the sales force}

Andrés Chávez

Laz Yangua Karen Denisse

Universidad Internacional del Ecuador, Ecuador

Autor por Correspondencia: anchavezer@internacional.edu.ec

Fecha de recepción: 28 de Febrero de 2017 - Fecha de aceptación: 28 de Abril de 2017

Resumen: Las organizaciones deben tener presente que la fuerza de ventas es el elemento clave para poder aumentar los ingresos y mantener la rentabilidad, es decir, se considera a los vendedores como el pilar fundamental de la empresa. Si bien es cierto las ventas existen desde la era primitiva, y su etapa con más presencia se da en la cultura fenicia, poco a poco ha sido perfeccionada a tal punto de ser considerada un arte. El presente recoge las herramientas principales que fomentan la productividad de la fuerza de ventas, como la estructura y tamaño que debe poseer, logrando reducir costos; otro elemento importante es la motivación que cada miembro del equipo posea y la que sienta que la organización le brinda, como el tan llamado sistema de recompensas (bonificaciones por cumplimiento de ventas entre otros), sistema comúnmente utilizado en el liderazgo transaccional, sin embargo debe considerarse que el dinero no es el único factor motivacional para la fuerza de ventas, el sentido de pertenencia a la empresa puede resultar más influyente que el mismo dinero. El sistema de control (acompañamiento y evaluación) a los vendedores permite visualizar como es el rendimiento y alcance de los objetivos propuestos; y es en donde los supervisores o jefes de ventas deben enfocar sus herramientas de capacitación convirtiendo las carencias o debilidades en puntos de fortaleza para alcanzar el objetivo en común. Palabras claves: fuerza de ventas; motivación; empoderamiento

Abstract: Organizations must keep in mind that the sales force is the key element in order to increase revenues and maintain profitability, that is, sellers are considered the fundamental pillar of the company. While sales have existed since the early era and its stage with more presence is in the Phoenician culture, little by little has been perfected to the point of being considered an art. The present offers the main tools that promote the productivity of the sales force, such as the structure and size that must have, reducing costs. Another important element is the motivation that each member of the team possesses and that which he feels the organization offers him, such as the so-called reward system (bonuses for sales fulfillment among others), a system commonly used in transactional leadership. However, considering that money is not the only motivational factor for the sales force, the sense of belonging to the company may be more influential than the same money. The system of control (accompaniment and evaluation) to the sellers allows to visualize as it is the performance and scope of the proposed objectives; and is where supervisors or sales managers should focus their training tools turning the gaps or weaknesses into strength points to achieve the goal in common.

Key words: sales force; motivation; empowerment 


\section{Introducción}

Alguna vez se ha preguntado ¿cómo lograr mantener o aumentar la productividad de la fuerza de ventas? En la actualidad, conservar a los miembros del equipo de ventas, se ha convertido en un reto que todas las organizaciones deben atravesar, ya que al ser el elemento clave o esencial para mantener firmes los pilares de la empresa, generar relaciones rentables con los clientes y que la organización obtenga rentabilidad, es en ellos en los que se deben centrar las estrategias adecuadas para que desarrollen sentido de pertenencia hacia la empresa.

Es así que es muy común que el jefe o la dirección del departamento de ventas requiera herramientas más eficaces para lograr aumentar la productividad de sus vendedores, todo con el fin de obtener un equipo más sólido y comprometido con sus actividades asignadas con el propósito de lograr alcanzar el objetivo en común que poseen: "llegar al presupuesto asignado o meta".

El número de los integrantes del equipo de ventas y la manera en la que están asignados a un número determinado de clientes, zona o territorio influirá en los resultados obtenidos en las ventas al final del periodo establecido, sin embargo otro de los factores que pueden afectar la productividad y el desempeño de la fuerza de ventas, es la motivación y los beneficios que la empresa puede brindarles.

El presente tiene como objetivo ofrecer algunas estrategias básicas para mejorar o, mantener la productividad de sus vendedores, pero que son tan importantes como para olvidarlas, desde la estructura y organización de la fuerza de ventas en la que se ofrecen dos métodos posibles a aplicar; la motivación y capacitación constante a los vendedores, así como la evaluación, acompañamiento y empowerment de las actividades asignadas.

\section{Marco Teórico}

A inicios de la creación de la humanidad, empezó el mundo de las ventas pero de manera primitiva; transacciones comerciales se daban de tribu en tribu con el denominado trueque. Poco a poco la evolución tecnológica, y la variante dinámica del mercado dieron lugar al mejoramiento de las técnicas de ventas y a considerar importantes a los vendedores; tanto es así que hoy en día, el disminuir la rotación de los miembros del departamento de ventas, mejorar la productividad y generar relaciones rentables con los clientes han generado un gran reto, a los que las organizaciones deben enfrentarse.

Según León y Noelia en su revista Ciencias Sociales Vol. XIX: la fuerza de ventas es el capital humano que se encarga de establecer una relación cercana con los clientes, escuchando las necesidades y buscando soluciones a los problemas que tengan ellos, esto los convierte en el punto clave para mejorar los procesos organizacionales (2013, pág. 381).

El jefe de ventas se debe encargar de la motivación y capacitación constante de su equipo, escuchar sugerencias, problemas e inconformidades presentes, con el fin de poder resolverlos y facilitar el proceso de toma de decisiones. Si se forjan excelentes bases, con relación a la 
colaboración, trabajo en equipo, confianza entre los integrantes de la fuerza de ventas, el resultado será favorable para quienes conforman el grupo.

Concentrarse en diversas estrategias, factores o herramientas para que la dirección de ventas, logre mejorar o mantener la productividad de sus colabores, puede resultar tan extenso en estudio, sin embargo existen algunos factores que son básicos y que pueden lograr resultados favorables, como los que se detallan en los siguientes párrafos del presente.

\section{Estructura y tamaño de la fuerza de ventas}

El objetivo del diseño o estructura de una organización es dividir y coordinar las tareas de modo que los colaboradores alcancen de forma eficaz los objetivos que tienen en común (Johnston \& Marshall, 2009). Estructurar a la fuerza de ventas, por clientes, zonas geográficas o territorios y por producto, permite tener una mayor cobertura hacia los clientes, en donde cada uno será capaz de administrar, organizar y controlar la zona asignada, la frecuencia en la que visitará a sus clientes, reduciendo los costos de movilización, tiempo, esfuerzos innecesarios, etc.

Si se divide cualquier actividad asignando a cada una de ellas un especialista o encargado, permitirá que la eficiencia y productividad aplicada a dichas tareas, mejoren en gran magnitud (Smith, 1776). Una de las ventajas de estructurar a la fuerza de ventas en uno de los tipos antes mencionados, es que define las funciones de cada vendedor, permitiendo que la evaluación de desempeño sea más fácil de realizar.

Si se hace referencia a la estructura u organización de la fuerza de ventas, se quiere indicar que es: la asignación de una zona geográfica propia de un vendedor, quien será responsable y encargado de las ventas de los bienes tangibles e intangibles en esa zona de mercado, en la cual la empresa posee presencia (Kotler P., 2001).

Una vez que las organizaciones hayan estructurado la fuerza de ventas, se puede proceder a calcular el tamaño de la misma. La fuerza de ventas forma parte de los activos con más productividad y costos para la empresa, por lo tanto, un aumento en su número significa considerablemente el incremento no solo en ventas sino también en los costos a incurrir (Kotler \& Armstrong, 2003).

Existen dos métodos para determinar el número o tamaño idóneo de la fuerza de ventas, propuestos por Semlow y Talley.

\section{Método de desglose}

Este método se enfoca en obtener el número óptimo de vendedores considerando que debe existir una igualdad entre los costos y beneficios de la empresa (Semlow, 1959).

Su fácil y rápida realización es una de las ventajas de la aplicación de este método, sin embargo entre sus desventajas se puede mencionar la exclusión del potencial de cada vendedor, así como el de los mercados o territorios existentes o la rotación del personal de ventas. 
Si la organización decide emplear este método debe basarse en su fórmula:

$\mathrm{N}=\mathrm{S} / \mathrm{P}$

En donde:

$\mathrm{N}=$ Cantidad de personal de ventas que se necesita

$\mathrm{S}=$ volumen pronosticado de ventas

$\mathrm{P}=$ Productividad o venta estimada para cada vendedor

Para comprender el método por desglose se presenta el siguiente ejercicio:

Una empresa que ha pronosticado las ventas en $\$ 25.000$ y en la que espera que cada vendedor facture $\$ 8.000$. ¿Cuántos vendedores necesitan la empresa?

$\mathrm{N}=\mathrm{S} / \mathrm{P}$

$\mathrm{N}=25.000 / 8.000$

$\mathrm{N}=3$

En cuestión del ejercicio antes mencionado, la empresa para alcanzar el presupuesto de ventas planteado con $\$ 8.000$ a cada vendedor, necesitará de la ayuda de tres vendedores.

\section{Método de carga de trabajo}

Este método denominado también como método de agregación, considera que todo el personal de ventas debe cargar una cantidad igual de trabajo, tomando en cuenta el número de clientes, la frecuencia de las visitas que requerirá cada uno y la duración de cada visita, considerando tiempos de desplazamiento y de administración.

Se puede aplicar este modelo para determinar el tamaño de la fuerza de ventas, así como la asignación de un vendedor a cada cliente (Talley, 1961).

Para mayor comprensión de este método a continuación se detalla un ejemplo:

La empresa "XYZ" posee 600 clientes segmentados en tres tipos (A, B Y C), se considera que el tiempo de desplazamiento y administración es del 13\%, y las horas laborables son de 8 horas diarias por 5 días a la semana.

\begin{tabular}{|c|c|c|c|c|c|}
\hline Tipo de cliente & $\mathbf{N}^{\circ}$ de clientes & $\begin{array}{l}\text { Frecuencia de } \\
\text { visita al mes }\end{array}$ & $\begin{array}{l}\text { Tiempo de } \\
\text { visita al mes }\end{array}$ & $\begin{array}{l}\text { Cálculo } \\
\text { hora/mes }\end{array}$ & $\begin{array}{l}\text { Total } \\
\text { horas/mes }\end{array}$ \\
\hline A & 100 & 6 & 1,5 & $100 * 6 * 1,5$ & 900 \\
\hline B & 150 & 4 & 0,5 & $150 * 4 * 0,5$ & 300 \\
\hline C & 350 & 4 & 0,5 & $350 * 4 * 0,5$ & 700 \\
\hline TOTAL & 600 & & & & 1900 \\
\hline
\end{tabular}

Tiempo de desplazamiento y administración: $18 \%$

Horas de trabajo: 160 horas al mes

Horas efectivas (horas de trabajo mes/tiempo de desplaz. y admin.): $169-18 \%=138$

$\mathrm{N}^{\circ}$ de vendedores (total horas mes/horas efectivas): 1900/ 138= 14 VENDEDORES 
Este método es muy fácil de comprender, además de que permite a la empresa reconocer los distintos tipos o categorías de clientes que poseen, sin embargo, no considera que un cliente de la misma categoría demande diferentes tiempos en cuanto a frecuencia y duración de cada visitas, lo que puede influir en el número estimado de vendedores.

\section{Motivación}

Neil Lebovits al recibir la asignación de presidencia de Ajilon, una empresa de reclutamiento con sede en Nueva Jersey, se dio cuenta de que esta tenía algunos problemas graves en cuanto a la relación existente ente colaboradores y la empresa; la rotación del personal era alta, la moral y la productividad estaban bajas. Buscando medidas para combatir estos problemas ofreció una fiesta después del trabajo en la cual sólo cinco empleados de 50 acudieron, fue ahí cuando Lebovits se dio cuenta de que tenía que buscar medidas urgentes para mejorar la motivación de sus empleados, pero lamentablemente no contaba con los recursos económicos suficientes como para establecer un sistema de recompensas monetario. Decidió entonces buscar diferentes alternativas que no necesitaran de mucho dinero, empezó con ofrecer programas de capacitación interna acerca de diversos temas en los cuales sus empleados habían manifestado interés. Adicional coordinó reuniones mensuales con cada colaborador con el objetivo de analizar decisiones gerenciales desde diferentes puntos de vista.

Estableció una dirección de correo electrónico donde los colaboradores podían proponer ideas y sugerencias las cuales eran respondidas. Además, dio a cada empleado tres días libres al año sin pedir explicaciones. A raíz de la implementación de estos beneficios hacia sus empleados, la moral y productividad de los empleados se elevaron en forma impresionante. Los empleados de la empresa incluso enviaron mensajes a Lebovits mostrándose muy agradecidos y motivados por el hecho de sentirse llenos de energía y voluntad para realizar sus funciones (Robbins \& Coulter, 2005).

La motivación juega un rol importante en el desempeño de los vendedores; si bien es cierto la recompensación monetaria es una de los motores principales para mantener al equipo de ventas enfocado en la meta, ya sea porque al final del mes obtendrán bonos, incentivos, premios, etc.; no es el único elemento capaz de motivar al recurso humano de ventas. El grado o nivel de motivación, puede variar de individuo a individuo y depende de las situaciones en las que este se ve involucrado.

La motivación también tiene factores psicológicos que la mayoría de las empresas se olvidan considerar, y sin embargo son estos factores los que más beneficios generan. Y ¿cuáles son esos factores psicológicos?, el ego, sentido de pertenencia; aplicándolo a ejemplos de las actividades organizacionales cotidianas está el: felicitar públicamente a un vendedor por el cumplimiento de metas, así como tomar en cuenta las sugerencias que cada miembro del equipo de ventas tiene para mejorar los procesos de la organización.

Dado que los motivos personales y laborales de los empleados influyen en su productividad, una de las tareas de la alta dirección es canalizar de forma efectiva las cosas que los motivan para que alcancen las metas propuestas por la compañía (Slocum \& Hellriegel, 2009). En el estudio de la motivación, existen diferentes teorías capaces de abarcar y comprender 
los diferentes factores de la motivación humana y de la motivación en el trabajo, las mismas que serán descritas en los párrafos posteriores.

\section{Teoría Jerarquía de las necesidades de Maslow}

Los seres humanos poseen una escala de necesidades que deben cubrir, desde su base hasta la cima de la pirámide como lo afirma Maslow, es decir, cubrir las necesidades fisiológicas para poder alcanzar a cubrir las de autorrealización. Por lo descrito, se puede deducir que no todos los individuos están motivados a satisfacer las mismas necesidades, por lo que cada uno buscará satisfacer las necesidades que crean necesarias para alcanzar la cima. El ser humano es un ser lleno de necesidades y a menudo no alcanza un estado de completa satisfacción, o se satisface solo en breves períodos; una vez satisfecho un deseo, aparece otro en su lugar, y tan pronto como este sea atendido aparecerá otro en su lugar, situándose como primero y así sucesivamente (Maslow, 1991).

\section{Teoría de McClelland}

La motivación se refiere a los propósitos conscientes e inconscientes, pensamientos íntimos e inferencias relativas a partir de la observación de conductas (McClelland, 1989). Esta teoría estudia la motivación en tres aspectos, proporcionando una visión global acerca de los motivos humanos que influyen a que los seres humanos se sientan o estén motivados .Los tres aspectos son: logro (obtener éxito destacando en el camino de la excelencia), afiliación (la necesidad de interactuar socialmente) y el poder (autoridad, prestigio y estatus).

\section{Teoría del factor dual de Herzberg}

Esta teoría supone que el bienestar del trabajador está relacionado a dos factores: $\mathrm{Si}$ hablamos de los factores higiénicos según la teoría de Herzberg, la motivación de los empleados dependerá de los factores externos a las actividades que este realiza, es decir, las relaciones personales con los compañeros de trabajo, el ambiente laboral, la seguridad del puesto y las políticas de la organización son influyentes; mientras que los factores motivadores se refieren al reconocimiento, logros, incentivos, asignación de responsabilidades.

\section{Teoría X y Y de McGregor}

Según McGregor, en las organizaciones existen dos tipos o estilos de colaboradores: Teoría X ser humano que siente una repugnancia intrínseca hacia el trabajo y lo evitará siempre que pueda (McGregor, 1994) mientras que la Teoría Y supone que los seres humanos se sienten a gusto en la organización donde laboran, buscan asumir responsabilidades y buscan siempre lograr sus objetivos.

\section{Capacitación}

Mientras trata de expandir el tamaño actual del mercado, la empresa líder debe defender continuamente sus ventas con las diferentes estrategias que pueda emplear contra los ataques de sus rivales, es así que Coca Cola, debe cuidarse de Pepsi Cola (Kotler P. , 2002). 
Cuando un nuevo empleado ingresa a laborar en la organización, es común observar que el nuevo personal es inducido por una serie de lineamientos, procesos y cultura organizacional. En el caso de los miembros del departamento de ventas, ellos son capacitados acerca de cada detalle de los bienes que la empresa ofrece a sus clientes, los socios o empresas aliadas a la organización, la competencia, etc. Adicional se les otorga una breve escuela de ventas, con el fin de lograr que sus vendedores cumplan con los objetivos, incluso los vendedores más destacados y antiguos son partícipes de las escuelas de ventas y demás programas de capacitación planificada por la empresa.

La capacitación al personal de ventas, puede mejorar el rendimiento o la capacidad de desenvolvimiento de los vendedores, aportando grandes beneficios a la organización, y puede ser comprendida como aquel proceso educativo de corta duración, pero que puede lograr que las personas consigan los objetivos propuestos.

En el mundo de las ventas, es muy importante considerar que los vendedores deben estar en constante capacitación, debido a la dinámica cambiante del mercado, considerando los factores externos e internos de la empresa, ya que con el avance actual de la tecnología los clientes hoy en día son más exigentes, por lo que la fuerza de ventas debe estar en constante actualización de las técnicas y estrategias de ventas para un cierre efectivo de las negociaciones.

Y es que el proceso de capacitación, también permite a las organizaciones detectar las debilidades y fortalezas del capital humano, con la finalidad de convertir esas debilidades en fortalezas y explotar los puntos fuertes existentes. Como referencia para el tema de capacitación, está la "Universidad del Café", creada por la cadena Ecuatoriana Sweet \& Coffee, con el objetivo de enseñar a colaboradores actuales y a los posibles trabajadores, las diversas técnicas para preparar los 42 tipos de café que se ofrecen en todos sus establecimientos en la ciudad de Guayaquil, este programa de capacitación permite asegurar la calidad y el buen sabor del principal producto de la cadena (Diario El Universo, 2009).

Otro caso de éxito en cuanto a lo importante que es la capacitación del personal y que gracias a la innovación tecnológica se puede lograr, es el de la empresa Bayer Health Care Pharmaceuticals, en conjunto con Concentric RX, una agencia de marketing de servicios para el cuidado de la salud, crearon un juego de video de simulación para capacitar a su fuerza de ventas en un nuevo programa de marketing de medicamentos, el innovador juego de video de interpretación de roles creado por RX, llamado RepRace: TheBattlefor Office Supremacy, ofrecía a los vendedores de Bayer mucho más entrenamiento que la anticuada prueba de habilidades de opción múltiple. El juego fue creado para reactivar las ventas de un producto que estaba en etapa de madurez, el Betaseron, un tratamiento terapéutico para la esclerosis múltiple (EM). El objetivo del juego era encontrar una forma más innovadora para ayudar a que los vendedores de la compañía apliquen la información aprendida acerca del Betaseron mediante ventas reales y en situaciones que en las que se presentaran el manejo de objeciones. Además buscaba incrementar la participación de los vendedores mediante un aprendizaje y retroalimentación interactivos con resultados en tiempo real. El valor educativo y motivacional del juego permitió que la compañía midiera el desempeño individual y colectivo de los vendedores. Al final, Bayer calculó que el juego de simulación RepRace sirvió para incrementar la eficacia del equipo de ventas del Betaseron en un 20 por ciento.(Kotler \& Gary, 2012) 


\section{Acompañamiento y Empowerment}

Emplear tiempo en los vendedores, para la retroalimentación, supervisión y enseñanza de técnicas para solucionar problemas que puedan presentarse con los clientes, es un factor que permite a la fuerza de ventas sentirse motivadas y refleja de los seguidores hacía el líder.

Dirigir una fuerza de ventas incluye evaluación y supervisión de las actividades asignadas al equipo. La dirección de ventas debe conocer que hace su equipo con el objetivo de establecer sistemas de recompensas o de castigos, e incluso para poder ofrecer propuestas constructivas de mejoramiento (Stanton, Etzel, \& Walker, 2007).

El acompañamiento es una de las herramientas más eficaces para capacitar al equipo, sin embargo, es una de las más rechazadas por ambas partes, ya que el vendedor puede sentirse asfixiado debido a que siente que puede vender más sin la presencia y presión del jefe, y por parte del jefe, el miedo a ser juzgado sino logra cerrar la venta; pero no hay que alejarse del objetivo del acompañamiento ya que este permite visualizar las estrategias empleadas, los gestos corporales y las destrezas que poseen, y aprender de las habilidades del otro. El jefe o la dirección de ventas, debe ser equitativo en la supervisión y acompañamiento de su equipo, ya que, si lo hace a menudo, puede causar hostigamiento, disminuir las capacidades y destrezas de ventas cada su equipo, y la no supervisión puede generar graves problemas de libertad y falta de responsabilidad de los vendedores hacia la empresa (Thompson, 2006), lo que afectará en el cumplimiento de la meta asignada.

Para hablar de empowerment se expresa el siguiente ejemplo: El vendedor de la empresa $\mathrm{XYZ}$ tiene todas las reglas marcadas acerca de sus funciones de venta, sin embargo es un vendedor sin ningún tipo de autonomía ni poder de decisión, este estará llamando en todo momento al jefe para pedir autorización y ayuda por cualquier situación que se le presenta (García, 2009), sin embargo si a este tipo de vendedor se le ofrece la capacitación y el empowerment necesario, podrá realizar sus negociaciones sin necesidad de consultar al jefe en temas que el vendedor pueda manejar.

El empowerment otra herramienta poco utilizada, pero que resulta útil, ya que otorgar a los vendedores un poco de poder en la toma de decisiones permite agilizar procesos en la atención con los clientes y en los cierres de ventas. Además mejora la productividad y la calidad de los colabores del equipo de ventas. (Chiavenato, 2015)

Y es que el empowerment, también permite desarrollar nuevas ideas de negocio, como es el caso de la notas Post-it de la compañía multinacional 3M, en el año 1968 Spencer Silver, un investigador de la compañía 3M estaba buscando un nuevo adhesivo potente, pero sólo consiguió uno que pegaba poco, al cual lo desechó por no cumplir con el objetivo en mente. En 1974 Art Fry otro colaborador de la empresa 3M, mientras estaba en la iglesia intentó leer algunos salmos, marcados en su libro, pero siempre se le perdían los separadores de los libros. Ante esta situación recordó el pegamento que inventó Silver y que tal vez podía pegar los separadores con este pegamento. 
Al día siguiente, Fry hizo un primer intento, el cual no le dio resultados favorables; pero siguió insistiendo por el apoyo que le daban sus pares. Un día Fry le entregó un libro a su jefe, en el que había introducido uno de sus separadores. Cuando se lo devolvió, el jefe le había escrito algo encima de aquella primera nota Post-it. Hoy en día son un suministro de oficina indispensable. (Isaza, 2015)

\section{Conclusiones}

La estructura y organización del equipo de ventas, permite tener un mejor control en el desempeño de cada vendedor, adicional el conocer con exactitud el número de integrantes necesarios en el equipo de ventas, permite a la organización no incurrir en gastos innecesarios de personal adicional.

Resulta difícil lograr mantener un equipo de ventas motivado, y con mente firme en cumplir con el presupuesto de ventas asignado, cuando no existe una planificación en cuanto a estrategias y métodos para el sistema de recompensas. Es común pensar que el dinero es el único factor con el cual los empleados pueden realizar sus funciones de forma eficiente y eficaz, sin embargo, el ofrecer un ambiente laboral afable, brindar la oportunidad de planes de carrera en la empresa, e incluso reconocimientos por las actividades realizadas con éxito, pueden aportar beneficio en cuando a la productividad de los trabajadores.

Se concluye que la capacitación constante que deben recibir los vendedores, es imprescindible, ya que las técnicas de ventas son parte de las herramientas que tienen los vendedores para lograr el cierre de ventas.

\section{Recomendaciones}

Considerando la estructura y organización del equipo de ventas, es necesario decidir cuál es el método que más se ajusta al giro de negocio de la organización, teniendo en cuenta de que tanto el método de desglose como el de carga de trabajo, tienen su porcentaje de error. Adicional las capacidades y destrezas de cada vendedor difieren entre sí, y las exigencias y necesidades de los clientes de la empresa son distintas.

Escuchar y atender las ideas provenientes de los colaboradores, permitirá mejorar los procesos de la organización, e incluso ayudará a analizar y tomar decisiones gerenciales considerando distintas percepciones, de esta manera el personal aumentará el grado de pertenencia a la empresa, mejorando su productividad en las tareas asignadas.

Es importante mantener en constante capacitación al personal de ventas, en cuanto a técnicas y estrategias para lograr un cierre exitoso de ventas, así como atención al cliente y manejo de objeciones, debido a que, hoy en día los clientes son más exigentes y poseen todas las herramientas tecnológicas para decidir que comprar y que no, tarea que resulta más difícil para los vendedores en cuestión de persuasión.

\section{Bibliografía}


Chiavenato, I. (17 de Diciembre de 2015). Las ventajas del empowerment en las empresas. Destino Negocio.

Diario El Universo. (06 de Enero de 2009). Una enseñanza que tiene sabor y aroma. El Universo.

García, L. M. (2009). Ventas (Segunda ed.). Madrid: ESIC Editorial.

Isaza, J. J. (2015). Breve historia de las marcas: Post- It. Bien pensado.

Johnston, M. M., \& Marshall, G. W. (2009). Administración de Ventas (Novena ed.). México D.F., México: Interamericana Editores.

Kotler, P. (2002). Dirección de Marketing - Conceptos esenciales (Primera ed.). México: Pearson Educación.

Kotler, P. (2001). Mercadotecnia. México: Hall Hispanoamericana.

Kotler, P., \& Armstrong, G. (2003). Fundamentos de Marketing (Sexta ed.). México: Pearson Educación.

Kotler, P., \& Gary, A. (2012). Marketing (Decimocuarta ed.). México: Pearson Educación.

León, N. (2013). Fuerza de ventas determinante de la competitividad empresarial. Ciencias Sociales, XIX N² 381.

Maslow, A. (1991). Motivación y Personalidad (Tercera ed.). Madrid: Ediciones Díaz de Santos.

McClelland, D. (1989). Estudio de la motivación humana. Madrid: Narcea S.A.

McGregor, D. (1994). El lado humano de las organizaciones. Santafé de Bogotá: McGraw Hill.

Robbins, S. P., \& Coulter, M. (2005). Administración (Octava ed.). México: Pearson Educación.

Semlow, W. (1959). How many salesmen do you need? (Vol. XXXVII). New York: Harvard Business Review.

Slocum, \& Hellriegel. (2009). Comportamiento Organizacional (Doceava ed.). México: Cengage Learning Editores.

Smith. (1776). La riqueza de las naciones. London: London Edition.

Stanton, W. J., Etzel, M. J., \& Walker, J. B. (2007). Fundamentos de Marketing. Décima Cuarta, 546. México D.F.: McGraw-Hill/Interamericana Editores S.A.

Talley, W. (1961). How to design sales territories. New York: Journal of Marketing.

Thompson, I. (2006). Administración de la Fuerza de Ventas. Promonegocios. 